\title{
A violência obstétrica e suas múltiplas faces: análise dos possíveis impactos na saúde
}

\section{da mulher}

\section{Obstetric violence and its multiple faces: analysis of possible impacts on women's health}

La violencia obstétrica y sus múltiples caras: análisis de posibles impactos en la salud de las mujeres

Deyse Janiele Bernardo Oliveira

ORCID: https://orcid.org/0000-0002-1270-8431 Universidade Federal do Rio Grande do Norte, Brasil

E-mail: deyse.oliveiira@hotmail.com

Hugo Sarmento Gadelha ${ }^{1}$

ORCID: https://orcid.org/0000-0001-9414-0554 Universidad del Museo Social Argentino, Argentina

E-mail: hugoscurso@uol.com.br

Márcio Flávio Lins de Albuquerque e Souto ${ }^{2}$

ORCID: https://orcid.org/0000-0002-3799-4913

Universidade Federal da Paraíba, Brasil

E-mail:marciofls@gmail.com

Ícaro da Silva Gomes

ORCID: https://orcid.org/0000-0003-3070-5514

Faculdade de Ciências Médicas da Paraíba, Brasil

E-mail: icarosgomes@gmail.com

José Isaul Pereira

ORCID: https://orcid.org/0000-0002-5027-0434 Universidade Federal do Rio Grande do Norte, Brasil

E-mail: isaulpsico@hotmail.com

Kelly Bezerra de Oliveira

ORCID: https://orcid.org/0000-0002-4499-1980

Universidade Federal do Rio Grande do Norte, Brasil E-mail: kelly.bez.oliveira@hotmail.com

Rejane Silva dos Santos

ORCID: https://orcid.org/0000-0001-7637-3856

Universidade Potiguar, Brasil

E-mail: rejannysantos32@gmail.com

Agílio Tomaz Marques

ORCID: https://orcid.org/0000-0001-8364-5063

Universidade Federal de Campina Grande, Brasil

E-mail: agiliotomaz@hotmail.com

Rosana Santos de Almeida

ORCID: https://orcid.org/0000-0001-8097-1074

Universidade Federal de Campina Grande, Brasil

E-mail: rosana.santos@estudante.ufcg.edu.br

\section{Resumo}

Considerado como uma questão de saúde pública, a violência obstétrica caracteriza-se como toda e qualquer ação violenta praticada contra mulheres no momento de pré-parto, parto e puerpério, podendo ocorrer nos serviços de saúde público ou privado, sendo sua prática constatada por diferentes atos violentos, sendo os principais de caráter físico, psicológico e institucional. O presente estudo teve como objetivo analisar as diferentes formas de apresentação da violência obstétrica, assim como suas possíveis implicações na saúde da mulher. Para tal, o estudo desenvolveu-se com base na pesquisa bibliográfica, amparando-se em referenciais teóricos que abordassem a temática da violência obstétrica e os impactos na qualidade de vida e saúde da mulher. A partir da pesquisa, pode-se constatar a necessidade da reformulação de práticas nos serviços de assistência materno infantil, que visem à educação permanente e

\footnotetext{
${ }^{1}$ Doutorando pela Universidad del Museo Social Argentino.

2 Membro da Academia Paraibana de Letras Jurídicas - APLJ; Advogado Licenciado com experiência forense nas áreas cível e eleitoral, e Professor Federal Universitário Adjunto III.
} 
formação em saúde dos profissionais, buscando alcançar a prevenção ou mitigação da violência obstétrica, assim como a incorporação de práticas humanizadas no parto e nascimento.

Palavras-chave: Violência obstétrica; Humanização no parto; Saúde da mulher; Impactos na saúde.

\begin{abstract}
Considered as a matter of public health, obstetric violence is characterized as any and all violent actions practiced against women at the time of pre-partum, childbirth and puerperium, which can occur in public or private health services, and its practice is verified by different violent acts, the main ones being physical, psychological and institutional. This study aimed to analyze the different forms of presentation of obstetric violence, as well as its possible implications for women's health. To this end, the study was developed based on bibliographical research, supported by theoretical references that addressed the theme of obstetric violence and the impacts on women's quality of life and health. From the research, it can be seen the need to reformulate practices in maternal and child care services, aimed at continuing education and health training for professionals, seeking to achieve the prevention or mitigation of obstetric violence, as well as the incorporation of practices humanized in childbirth and birth.
\end{abstract}

Keywords: Obstetric violence; Humanization in childbirth; Women's health; Health impacts.

\title{
Resumen
}

Considerada como una cuestión de salud pública, la violencia obstétrica se caracteriza como todas y cada una de las acciones violentas practicadas contra la mujer en el momento del preparto, parto y puerperio, que pueden ocurrir en los servicios de salud públicos o privados, y su práctica es verificada por diferentes actos violentos, siendo los principales físicos, psicológicos e institucionales. Este estudio tuvo como objetivo analizar las diferentes formas de presentación de la violencia obstétrica, así como sus posibles implicaciones para la salud de la mujer. Para ello, el estudio se desarrolló a partir de una investigación bibliográfica, sustentada en referencias teóricas que abordaron el tema de la violencia obstétrica y los impactos en la calidad de vida y salud de las mujeres. De la investigación se desprende la necesidad de reformular prácticas en los servicios de atención maternoinfantil, orientadas a la educación y formación continua en salud de los profesionales, buscando lograr la prevención o mitigación de la violencia obstétrica, así como la incorporación de prácticas humanizadas. en el parto y el parto.

Palabras clave: Violencia obstétrica; Humanización en el parto; La salud de la mujer; Impactos en la salud.

\section{Introdução}

Por décadas, a história da assistência ao parto e nascimento no Brasil é marcada por significativas mudanças, impulsionadas pelos movimentos sociais da época que lutavam contra a patologização dos processos fisiológicos do parto e nascimento, a medicalização do corpo feminino, a despersonalização pela perda da autonomia da parturiente e das inúmeras intervenções desnecessárias e invasivas na mulher e no bebê (Sena \& Tesser, 2017; Andrade \& Aggio, 2016).

Nesse contexto assistencial, a mulher era retirada do seu papel de protagonista do parto, tornando-se elemento secundário desse processo, submetida à dinâmica hospitalar e obrigada a seguir as ordens e protocolos institucionais, tendo seu livre direito de decidir sobre seu corpo e seus processos reprodutivos violentamente silenciado pelos profissionais de saúde, cercados de poder e de autoridade técnico-científica (Jardim \& Modena, 2018).

O termo "violência obstétrica" é considerado relativamente novo, sendo um assunto abordado inicialmente na década de 1950, através de uma publicação em uma revista dos Estados Unidos, a Ladies Home Journal, voltada ao público feminino. A matéria foi intitulada de "Crueldades nas maternidades" e retratava os maus tratos vivenciados por mulheres gestantes, submetidas a procedimentos violentos nos serviços de saúde (Diniz et al., 2015).

No Brasil, o tema ganhou visibilidade na década de 1980 em decorrência das fortes reivindicações dos movimentos sociais e grupos feministas da época, através de discussões pautadas na humanização do parto e nascimento a partir da redução da violência obstétrica e da garantia do direito à liberdade de escolha e fortalecimento do protagonismo da mulher no parto (D’Aquino, 2016).

Diversos autores estudam a violência obstétrica a partir de uma concepção de gênero, uma vez que este fenômeno caracteriza-se como uma forma de violência contra a mulher e, segundo Henriques (2021) vêm ganhando repercussão como um desafio de saúde pública no cenário brasileiro, dado que o número da prevalência da violência obstétrica no Brasil varia entre $18,3 \%$ a $44,3 \%$. 
Nesse sentido, por ser considerado um fenômeno multifatorial, a violência obstétrica tem natureza ampla, podendo ser evidenciada de diferentes formas, sendo as mais comuns no contexto obstétrico as violências de caráter físico, psicológico e institucional (Diniz et al., 2015).

Compreendendo o exposto, o presente estudo objetiva analisar as diferentes formas de apresentação da violência obstétrica, assim como suas possíveis implicações na saúde da mulher, visando à ampliação da temática e o fortalecimento da humanização do parto e nascimento.

Desse modo, faz-se relevante pesquisar sobre o tema, tendo em vista que trata-se de um fenômeno comumente evidenciado nos contextos dos serviços de saúde, e apesar de ser um tema debatido há décadas, a vasta produção de estudos encontrados na literatura científica reafirmam a importância de discutir a violência obstétrica em todas as suas múltiplas faces, visando a elaboração de medidas e estratégias à partir da compreensão dos impactos na saúde da mulher.

Ademais, no que refere-se à metodologia adotada, tratar-se-á de uma pesquisa de cunho bibliográfico, a partir de consultas realizadas em artigos de periódicos científicos, livros, teses, dissertações de mestrado e documentos emitidos pela Organização Mundial da Saúde, tendo como descritores: Violência Obstétrica, Humanização no parto, Saúde da mulher, Impactos na saúde, a partir de pesquisas nas principais bases de dados científicos, como a Scienific Eletronic Library Online (SciELO), Biblioteca Virtual em Saúde (BVS) e o Google Acadêmico. Inicialmente, foi realizado a pré-seleção dos artigos, tendo como critério de inclusão àqueles que abordassem a temática e fossem produzidos entre os anos de 2011 a 2021. Em seguida, realizar-se-á uma leitura crítica reflexiva a fim de selecionar os assuntos mais relevantes.

\section{Desenvolvimento}

A gestação, o parto e o nascimento normalmente são eventos marcantes e significativos para a mulher e seus familiares, contudo, muitas vezes esse momento é carregado por lembranças de medo, tristeza e traumas decorrentes de uma assistência marcada por vivências violentas e invasivas (Medeiros et al., 2016).

Nesse sentido, a violência obstétrica é reconhecida pela Organização Mundial da Saúde (2014) como uma questão de saúde pública, podendo ser conceituado como todo e qualquer ato violento, abusivo, desrespeitoso e de maus-tratos durante a gestação, parto e puerpério, assim como em situações que envolvem perdas gestacionais e assistências ao ciclo reprodutivo.

Ainda, de acordo com estudo de base populacional realizado no país, o índice de prevalência da violência obstétrica no Brasil tem variado entre 18,3\% a 44,3\%, e mesmo com a alta prevalência, observa-se a escassez de estudos epidemiológicos nacionais sobre a temática, como também poucos estudos que evidenciam as consequências dos atos de Violência Obstétrica na saúde da mulher (Henriques, 2021).

Dentro dessa perspectiva, diversos autores se propuseram a investigar a violência obstétrica a partir de uma abordagem multidimensional e multifatorial, compreendendo que trata-se de um fenômeno que se apresenta de diferentes formas, tais quais: violência física, violência institucional, violência psicológica e verbal, violência moral e violência sexual (Carvalho \& Brito, 2016; Souza et al., 2016; Martins \& Barros, 2016).

Desse modo, o de caráter institucional normalmente é o primeiro tipo de violência sofrido pela gestante e seus familiares, uma vez que constitui-se como atos ou modelos organizativos dos serviços que prejudiquem à assistência integral à mulher ou torne inacessível a garantia dos seus direitos assegurados por lei (Souza, Rattner, Gubert, 2017; Marrero \& Bruggemann, 2018).

Segundo Martins e Barros (2016) a violência institucional pode ser exposto pela falta de estrutura física adequada nos serviços, recursos físicos e humanos insuficientes, como também a proibição da presença de doulas na assistência ao parto, a 
privação do direito ao acompanhante na sala de parto ou a proibição dos pais na sala de pré-parto, por alegar que a figura de um homem perturbará a privacidade das demais parturientes.

Ainda, outro tipo de violência é a de caráter físico, descrita na literatura como atos que ocorrem na assistência ao parto, sendo comumente observado através da realização de procedimentos sem o devido consentimento da mulher ou a realização de condutas sem evidências científicas, como por exemplo, a cesariana eletiva; a manobra de kristeller, que é caracterizada pela pressão na parte superior do útero da mulher a fim de auxiliar na saída do bebê; a episiotomia de rotina; a litotomia, que trata-se da posição inadequada e desfavorável ao nascimento do bebê, entre outros (Martins \& Barros, 2016).

Ademais, a violência psicológica e verbal soma-se também, como formas de violência frequentemente evidenciadas no ambiente dos serviços de saúde, podendo ser identificadas através de comportamentos como abuso da autoridade, hostilidade, ironias, gritos, ameaças, chantagens, comentários desrespeitosos, humilhações, entre outros, corroborando diretamente no desajuste psicológico e emocional da mulher, despertando-a sentimentos como: tristeza, culpabilização, medo, inferioridade, etc. (Martins \& Barros, 2016).

A violência moral normalmente está associada às condutas dos profissionais que anulam o papel da mulher enquanto protagonista do parto e transferem essa função ao médico, cabendo a este a responsabilidade de decidir sobre os procedimentos utilizados e a conduta ativa do processo, reforçando a ideia de mulheres alienadas, despersonalizadas, com suas identidades anuladas e diminuídas a meros números de prontuários ou leitos de enfermarias, corroborando para a desvalorização da autonomia da mulher de decidir sobre seu corpo e seus processos reprodutivos (Assis, Meurer, \& Delvan, 2021; Martins \& Barros, 2016).

Por último, e não menos importante, temos a violência sexual, que apesar de não ser tão retratada na literatura como os outros tipos de violência supramencionados, pode ser conceituado como todo comportamento que incida sobre o senso de integridade sexual e reprodutiva da mulher, como por exemplo, assédio; exames de toques invasivos, sem consentimento ou de forma constante e agressivos; lavagem intestinais, entre outros (Jardim \& Modena, 2018).

Considerando a violência obstétrica em seu caráter multifatorial, sabe-se que esta aflige a mulher de diferentes modos e as implicações desses atos de violência podem ser visualizados desde sequelas físicas, com a presença de dores após procedimento desnecessário, como também sequelas emocionais, com o surgimento de traumas psicológicos ocasionados pela vivência de eventos causadores de dores e sofrimento (Rocha \& Grisi, 2017).

De acordo com um inquérito nacional realizado nos anos de 2011-2012, observa-se os números alarmantes de 56,6\% relacionados aos brasileiros que nasceram por cesarianas na rede pública e $90 \%$ na rede privada. Das mulheres que tiveram parto vaginal, $53,5 \%$ sofreram episiotomia e 36,1\% relataram manobra de Kristeller. Em relação ao uso de ocitocina no trabalho de parto foram identificados que 36,4\% receberam o medicamento para induzir ou acelerar o processo e $39,1 \%$ sofreram aminiotomia (Leal et al., 2012).

Dito isto, Oliveira e Maia (2020) e Costa et al. (2015) destacam em seus estudos as implicações advindas do uso rotineiros da episiotomia, que trata-se de um procedimento cirúrgico que consiste em uma incisão no períneo para facilitar a saída do bebê, com indicação em casos de estrita necessidade, uma vez que as dores resultantes dessa prática perpetuam por um tempo e interferindo em atividades cotidiana durante o pós-parto, como sentar ou caminhar, e ainda, muitas vezes transportando a puérpera para um lugar de lembrança desagradáveis vivenciadas no parto.

Nessa perspectiva, estudos como o de Diniz et al. (2015) enfatizam que a violência obstétrica tem implicações diretas sobre a morbimortalidade materna, destacando os riscos associados aos eventos adversos decorrentes de intervenções consideradas potencialmente agressivas e danosas no parto vaginal, como o uso de episiotomia, a manobra de kristeller, uso de fórceps e uso não regulado da ocitocina para induzir ou acelerar o parto. 
A hostilidade, negligência, julgamentos e retardo no atendimento ofertado às mulheres nos serviços de saúde, principalmente em contexto de abortamento são identificados como um tipo de violência obstétrica de potencial risco de morbimortalidade materna, uma vez que a demora em responder as demandas ou na prestação da assistência, poderão resultar em danos severos à saúde da mulher (Diniz et al., 2015).

Além das implicações fisiológicas, as intervenções decorrentes de práticas violentas também resultam em impactos na saúde mental das mulheres, tendo em vista que a vivência de situações desagradáveis durante o parto poderá resultar no surgimento de sintomas depressivos, traumas psicológicos, receio em ter uma outra gestação, disfunções sexuais, entre outros (Soares et al., 2015).

Nesta esteira, a associação entre a depressão pós-parto e a violência institucional sofrida pela mulher durante a assistência ao parto e nascimento também são tratados nos estudos de Souza, Rattner e Gubert (2017), que destacam a falta de humanização no atendimento como fatores de riscos.

Desta maneira, faz-se imprescindível a formulação de medidas que auxiliem na redução de práticas violentas no contexto da assistência obstétrica, uma vez que são diversos os impactos na saúde dessas mulheres e seus familiares, resultando em danos físicos, psicológicos e na sua qualidade de vida. Sendo assim, cabe aos serviços propor ações para humanizar a assistência ao parto e nascimento, impulsionando que a mulher desenvolva sua autonomia, cabendo aos profissionais de saúde, acolher, respeitar e apoiar suas decisões (Araújo et al., 2020; Barbosa, Fabbro, \& Machado, 2017).

\section{Considerações Finais}

Percebe-se, mediante considerações abordadas durante o texto, que a que a violência obstétrica trata-se de um fenômeno complexo, multidimensional e multifatorial, acometendo a mulher em diferentes modos de apresentação e reverberando em sérios impactos na sua saúde física e mental.

Dito isto, reitera-se a importância de ofertar um cuidado humanizado, respeitoso e empático para essas mulheres, haja visto que o ambiente onde tem o papel de propiciar uma assistência ao parto e nascimento, acaba, muitas vezes, tornando-se um depósito de lembranças de maus-tratos e violência.

Nesse sentido, faz-se necessário a reformulação de práticas nos serviços de saúde, investir em intervenções que visem à educação permanente e a formação em saúde dos profissionais do serviço, visando a incorporação de condutas baseadas em evidências científica e embasadas na Política Nacional de Humanização e no Programa de Humanização no pré-natal e nascimento, a fim de prevenir e mitigar a violência obstétrica.

Ainda, cabe a estruturação e desenvolvimento de ações voltadas à informação das mulheres e seus familiares em relação à identificação de práticas consideradas como violência obstétrica, seus direitos instituídos em lei, os dispositivos de suporte e apoio à mulher e instigar o seu papel de protagonismo no processo de parturição, implicando no fortalecimento da autonomia das usuárias e seus familiares ante ao parto e nascimento.

Por tudo isto, entende-se que o estudo alcançou os objetivos propostos, salientando a importância da produção de pesquisas adicionais na área, que fomente a discussão sobre as possíveis estratégias para a humanização do cuidado na assistência materno infantil.

Por fim, como sugestão para continuidade de discussões nesse sentido, analisar, à luz da realidade fática, de que modo as facetas da violência obstétrica se apresenta, contribuirá para o fortalecimento e planejamento de estratégias de atenção a este público, assim como desenhar e formular novos e mais efetivos caminhos em direção à efetivação dos direitos inerentes aos idosos. 


\section{Referências}

Andrade, B. P., \& Aggio, C. M. (2014). Violência obstétrica: a dor que cala. In Anais do III Simpósio Gênero e Políticas Públicas, Londrina: Universidade Estadual de Londrina. ISSN: 2177-8248. http://www.uel.br/eventos/gpp/pages/arquivos/GT3_Briena\%20Padilha\%20Andrade.pdf

Araújo, B. C. et al. (2020). Um olhar sobre a violência obstétrica / A look at obstetric violence. Brazilian Applied Science Review, [S.L.], 4(6). Brazilian Applied Science Review. http://dx.doi.org/10.34115/basrv4n6-058.

Assis, K. G. de; Meurer, F. (2020). Repercussões emocionais em mulheres que sofreram violência obstétrica. Psicologia Argumento, [S.L.], 39(103). Pontificia Universidade Catolica do Parana - PUCPR. http://dx.doi.org/10.7213/psicolargum.39.103.ao07.

Barbosa, L. de C., Fabbro, M. R. C., \& Machado, G. P. dos R. (2017). Violência obstétrica: revisão integrativa de pesquisas qualitativas. Rev.enferm., Bogotá, $35(2)$.

Carvalho, I. da S., \& Brito, R. S. de. (2017). Formas de violência obstétrica vivenciadas por puérperas que tiveram parto normal. Enfermería Global, [S.L.], 16(3). Servicio de Publicaciones de la Universidad de Murcia. http://dx.doi.org/10.6018/eglobal.16.3.250481.

Costa, L. M. et al. (2015). Episiotomia no parto normal: incidência e complicações. Revista Cultural e Científica, UNIFACEX, 13(1).

D'Aquino, L. S. (2016). O movimento de humanização do parto como movimento social e suas semelhanças com os recentes protestos que tomaram o mundo. Revista Direitos Sociais e Políticas Públicas, [s. l], 4(1).

Diniz, S. G. et al. (2015). ABUSE AND DISRESPECT IN CHILDBIRTH CARE AS A PUBLIC HEALTH ISSUE IN BRAZIL: origins, definitions, impacts on maternal health, and proposals for its prevention. Journal Of Human Growth And Development, [S.L.], 25(3). Faculdade de Filosofia e Ciências. http://dx.doi.org/10.7322/jhgd.106080.

Henriques, T. (2021). Violência obstétrica: um desafio para saúde pública no Brasil. Páginagrená, Rio de Janeiro.

Jardim, D. M. B., \& Modena, C. M. (2018). Obstetric violence in the daily routine of care and its characteristics. Revista Latino-Americana de Enfermagem, [S.L.], v. 26.

Leal, M. do Carmo et al. (2012). Birth in Brazil: national survey into labour and birth. Reproductive Health, [S.L.], 9(1). Springer Science and Business Media LLC. http://dx.doi.org/10.1186/1742-4755-9-15.

Marrero, L., \& Bruggemann, O. M. (2018). Violência institucional durante o processo de parturição no Brasil: revisão integrativa. Rev. Bras. Enferm. Brasília, $71(3)$.

Martins, A. de C., \& Barros, G. M. (2016). Você vai dar à luz na dor? Revisão integrativa da violência obstétrica em unidades públicas brasileiras. Rev. dor, São Paulo, 17(3).

Medeiros, N. C. M. et al. (2016). Violência obstétrica: percepções acerca do parto normal. Rev. Tem em Saúde, João Pessoa, 16(3).

Rocha, M. J., \& Grisi, E. P. (2017). Violência Obstétrica e suas Influências na Vida de Mulheres que Vivenciaram essa Realidade. Revista Multidisciplinar e de Psicologia, [s. l], 11(38).

Oliveira, E. L. de., Maia, E. M. C. (2020). Implicações da violência obstétrica para as mulheres durante o parto eutócico hospitalar: uma revisão sistemática. Temas em Saúde, [S.L.], 20(1). Even3. http://dx.doi.org/10.29327/213319.20.1-16.

Organização Mundial da Saúde. (2014). Prevenção e eliminação de abusos, desrespeito e maus-tratos durante o parto em instituições de saúde. Genebra: WHO. http://apps.who.int/iris/

Sena, L. M., \& Tesser, C. D. (2016). Violência obstétrica no Brasil e o ciberativismo de mulheres mães: relato de duas experiências. Interface - Comunicação, Saúde, Educação, [S.L.], 21(60). FapUNIFESP (SciELO). http://dx.doi.org/10.1590/1807-57622015.0896.

Soares, P. B. et al. (2015). Violência obstétrica e suas implicações. Revista Norte Mineira de Enfermagem.

Souza, A. B. et al. (2016). Fatores associados à ocorrência de violência obstétrica institucional: revisão integrativa da literatura. Rev. Ciênc. Méd., Campinas, $25(3)$.

Souza, K. J. de., Rattner, D., \& Gubert, M. B. (2017). Institutional violence and quality of service in obstetrics are associated with postpartum depression. Revista de Saúde Pública, [S.L.], v. 51. FapUNIFESP (SciELO). http://dx.doi.org/10.1590/s1518-8787.2017051006549. 